PROCEEDINGS OF THE

AMERICAN MATHEMATICAL SOCIETY

Volume 127, Number 5, Pages 1473-1482

S 0002-9939(99)05016-9

Article electronically published on January 29, 1999

\title{
COMPLETENESS OF EIGENVECTORS OF GROUP REPRESENTATIONS OF OPERATORS WHOSE ARVESON SPECTRUM IS SCATTERED
}

\author{
SEN-ZHONG HUANG
}

(Communicated by David R. Larson)

\begin{abstract}
We establish the following result.
Theorem. Let $\alpha: G \rightarrow \mathcal{L}(X)$ be a $\sigma\left(X, X_{*}\right)$ integrable bounded group representation whose Arveson spectrum $\operatorname{Sp}(\alpha)$ is scattered. Then the subspace generated by all eigenvectors of the dual representation $\alpha^{*}$ is $w^{*}$ dense in $X^{*}$. Moreover, the $\sigma\left(X, X_{*}\right)$ closed subalgebra $W_{\alpha}$ generated by the operators $\alpha_{t}$ $(t \in G)$ is semisimple.

If, in addition, $X$ does not contain any copy of $c_{0}$, then the subspace spanned by all eigenvectors of $\alpha$ is $\sigma\left(X, X_{*}\right)$ dense in $X$. Hence, the representation $\alpha$ is almost periodic whenever it is strongly continuous.
\end{abstract}

\section{SPECTRAL THEORY FOR INTEGRABLE BOUNDED GROUP REPRESENTATIONS}

Throughout this paper $G$ will denote a locally compact abelian (LCA) group with identity $e$ and $\widehat{G}$ will denote the dual group of $G$. The multiplication on LCA groups will be written by addition. Let $L^{1}(G)$ (resp. $M(G)$ ) be the usual group algebra (resp. measure algebra) with convolution as product operation. We refer to [11] or [21] for basic knowledge of Harmonic Analysis on LCA groups.

Given a complex Banach space $X$, let $\mathcal{L}(X)$ be the Banach algebra of all bounded linear operators on $X$. Take a LCA group $G$. A bounded group representation $\alpha$ of $G$ on $X$ is a mapping $\alpha: G \rightarrow \mathcal{L}(X)$ satisfying the following properties:

(a) Group property: $\alpha_{e}=I_{X}$ the identity operator on $X$ and $\alpha_{s+t}=\alpha_{s} \alpha_{t}$ for all $s, t \in G$;

(b) Boundedness: $\|\alpha\|:=\sup _{t \in G}\left\|\alpha_{t}\right\|<\infty$.

Moreover, $\alpha$ is called strongly (resp. weakly) continuous if for each $x \in X$ the mapping $t \mapsto \alpha_{t} x$ is norm (resp. weakly) continuous. We need a further notion.

Definition 1.1. A bounded group representation $\alpha: G \rightarrow \mathcal{L}(X)$ of $G$ on $X$ is called integrable if there exists a subspace $X_{*} \subset X^{*}$ satisfying the following

Received by the editors September 1, 1997.

1991 Mathematics Subject Classification. Primary 47A67, 47A10.

Key words and phrases. Spectrum of group representation, almost periodicity.

(C)1999 American Mathematical Society 
requirements:

(i) $X_{*}$ is a norm determining subspace of $X^{*}$, i.e., the following

$$
\|x\|_{1}:=\sup \left\{|\rho(x)|: \rho \in X_{*},\|\rho\| \leq 1\right\}, \quad x \in X,
$$

defines an equivalent norm on $X$;

(ii) The group representation $\alpha$ is $\sigma\left(X, X_{*}\right)$ continuous and for each $\mu \in M(G)$ there exists an operator $\tilde{\alpha}_{\mu} \in \mathcal{L}(X)$ such that

$$
\rho\left(\tilde{\alpha}_{\mu} x\right)=\int_{G} \rho\left(\alpha_{t} x\right) d \mu(t) \text { for all }(x, \rho) \in X \times X_{*} .
$$

In this case we say that $\alpha$ is $\sigma\left(X, X_{*}\right)$ integrable and the operators $\tilde{\alpha}_{\mu}$ are written as

$$
\tilde{\alpha}_{\mu}=\sigma-\int_{G} \alpha_{t} d \mu(t), \quad \mu \in M(G) .
$$

It is easily verified that the extension $\tilde{\alpha}: M(G) \rightarrow \mathcal{L}(X)$ is a bounded algebra homomorphism, i.e.,

$$
\tilde{\alpha}_{\mu * \nu}=\tilde{\alpha}_{\mu} \tilde{\alpha}_{\nu}, \quad \mu, \nu \in M(G) .
$$

Moreover, $\alpha_{t}=\tilde{\alpha}_{\delta_{t}}(t \in G)$, where $\delta_{t}$ is the Dirac measure at the point $t$.

In the sequel $\tilde{\alpha}$ will denote the algebra homomorphism which is obtained by integrating a group homomorphism $\alpha$.

That all weakly continuous bounded group representations are integrable is wellknown; see [1].

Let $\alpha: G \rightarrow \mathcal{L}(X)$ be an integrable bounded group representation. For $f \in$ $L^{1}(G)$, let $\tilde{\alpha}_{f}$ be the image of the measure $d \mu_{f}(t):=f(t) d t$ under $\tilde{\alpha}$. It is clear that $\tilde{\alpha}: L^{1}(G) \rightarrow \mathcal{L}(X)$ is also a bounded algebra homomorphism. Let $I_{\alpha}:=\{f \in$ $\left.L^{1}(G): \tilde{\alpha}_{f}=0\right\}$. The Arveson spectrum of $\alpha$, denoted by $\operatorname{Sp}(\alpha)$, is defined as the hull of $I_{\alpha}$, i.e.,

$$
\operatorname{Sp}(\alpha):=\left\{\gamma \in \widehat{G}: \hat{f}(\gamma)=0 \text { for all } f \in I_{\alpha}\right\} .
$$

For $x \in X$, let $I_{x}:=\left\{f \in L^{1}(G): \tilde{\alpha}_{f} x=0\right\}$ and define

$$
\operatorname{Sp}_{\alpha}(x):=\left\{\gamma \in \widehat{G}: \hat{f}(\gamma)=0 \text { for all } f \in I_{x}\right\}
$$

to be the spectrum of $\alpha$ at the point $x$. For a closed subset $\Lambda$ of $\widehat{G}$, define

$$
X^{\alpha}(\Lambda):=\left\{x \in X: \operatorname{Sp}_{\alpha}(x) \subseteq \Lambda\right\}
$$

to be the spectral subspace corresponding to $\Lambda$. A $\gamma \in \widehat{G}$ is called an eigenvalue if the eigenspace $\left\{x \in X: \alpha_{t} x=\gamma(t) x \forall t \in G\right\}$ is non-trivial. Eigenvectors are defined similarly.

We need the following basic facts established by the author in [14, Chapter I], cf. [1], [3], [4] and [7]. A complete summary of [14] appeared in "Dissertation Summary in Mathematics" 1 (1996), 171-178.

Theorem 1.2. Assume $X \neq\{0\}$. Let $\alpha: G \rightarrow \mathcal{L}(X)$ be a $\sigma\left(X, X_{*}\right)$ integrable bounded group representation. Then,

(i) The Arveson spectrum $\mathrm{Sp}(\alpha)$ is a non-empty closed subset of $\widehat{G}$. Assume $\gamma \in \widehat{G}$. Then, $\gamma \in \operatorname{Sp}(\alpha)$ if and only if there exists a net $\left(x_{i}\right)$ of norm-one vectors in $X$ such that $\left\|\alpha_{t} x_{i}-\gamma(t) x_{i}\right\| \rightarrow 0(i \rightarrow \infty)$ uniformly for $t$ in every compact subset of $G$. 
(ii) The group representation $\alpha$ is norm continuous if and only if its Arveson spectrum $\operatorname{Sp}(\alpha)$ is compact.

(iii) Assume that $\operatorname{Sp}(\alpha)$ is decomposed into disjoint closed subsets $E$ and $F$ where $E$ is compact. Then there exists a projection $P \in\left\{\tilde{\alpha}_{f}: f \in L^{1}(G)\right\}$ such that

$$
X^{\alpha}(E)=P X \quad \text { and } \quad X^{\alpha}(F)=\left(I_{X}-P\right) X .
$$

For the subspace representations $\alpha \circ P$ and $\alpha \circ\left(I_{X}-P\right)$ obtained by restricting $\alpha$ in $P X$ and $\left(I_{X}-P\right)$ there holds

$$
\operatorname{Sp}(\alpha \circ P)=E \text { and } \operatorname{Sp}\left(\alpha \circ\left(I_{X}-P\right)\right)=F .
$$

(iv) The following spectral mapping theorem holds:

$$
\sigma\left(\alpha_{t}\right)=\overline{\{\gamma(t): \gamma \in \operatorname{Sp}(\alpha)\}} \text { for all } t \in G .
$$

(v) Let $K(G):=\left\{f \in L^{1}(G): \hat{f}\right.$ has compact support $\}$. Then, the subspace generated by all vectors $\tilde{\alpha}_{f} x(f \in K(G)$ and $x \in X)$ is $\sigma\left(X, X_{*}\right)$ dense in $X$.

(vi) If $G:=\mathbb{Z}$ and $T \in \mathcal{L}(X)$ is the generator of the representation $\alpha: \mathbb{Z} \rightarrow \mathcal{L}(X)$, then $\operatorname{Sp}(\alpha)=\sigma(T)$, where $\sigma(T)$ is the spectrum of the operator $T$.

(vii) If $G:=\mathbb{R}$ and $A$ is the infinitesimal generator of the one-parameter group $\left(\alpha_{t}\right)_{t \in \mathbb{R}}$, then $\operatorname{Sp}(\alpha)=i \sigma(A)$, where $\sigma(A)$ is the spectrum of the closed operator $A$.

As a remark we point out that a weakly continuous bounded group representation $\alpha: G \rightarrow \mathcal{L}(X)$ is in fact strongly continuous. To see this, take $f \in K(G)$ and let $X_{f}:=\overline{\tilde{\alpha}_{f} X}$. Consider the restriction of $\alpha$ in $X_{f}$, denoted by $\beta$. To estimate the spectrum of $\beta$, let $\gamma \in \widehat{G} \backslash \operatorname{supp} \hat{f}$. Then, by the regularity of $L^{1}(G)$ there exists $g \in L^{1}(G)$ such that $\hat{g}(\gamma)=1$ and $\operatorname{supp} \hat{g} \subseteq \widehat{G} \backslash \operatorname{supp} \hat{f}$. It follows that $g * f=0$ and thus $\tilde{\beta}_{g} \tilde{\alpha}_{f}=\tilde{\alpha}_{g} \tilde{\alpha}_{f}=\tilde{\alpha}_{g * f}=0$. This implies by definition of $\operatorname{Sp}(\beta)$ that $\gamma \notin \operatorname{Sp}(\beta)$ and thus $\operatorname{Sp}(\beta)$ is contained in the compact subset supp $\hat{f}$. By Theorem 1.2 (ii) $\beta$ is norm continuous. This implies that the function $t \mapsto \alpha_{t} x$ is norm continuous for each $x \in X$ which can be written as $x=\tilde{\alpha}_{f} y$ for some $y \in X$ and $f \in K(G)$. As claimed by Theorem $1.2(\mathrm{v})$, such vectors generate a weakly dense and hence norm dense subspace of $X$. In conclusion, $\alpha$ is strongly continuous.

We need also two auxiliary results.

Proposition 1.3. Let $\alpha: G \rightarrow \mathcal{L}(X)$ be an integrable bounded group representation. Assume $\gamma$ to be an isolated point of $\operatorname{Sp}(\alpha)$. Then, there exists a projection $0 \neq P_{\gamma} \in\left\{\tilde{\alpha}_{f}: f \in L^{1}(G)\right\}$ such that

$$
\alpha_{t} P_{\gamma}=\gamma(t) P_{\gamma} \text { for all } t \in G .
$$

In particular, $\gamma$ is an eigenvalue of $\alpha$.

Proof. Let $P_{\gamma}$ be the spectral projection corresponding to the set $\{\gamma\}$ for which we have

$$
\operatorname{Sp}\left(\alpha \circ P_{\gamma}\right)=\{\gamma\}
$$

The existence of $P_{\gamma}$ is guaranteed by Theorem 1.2 (iii). Applying Theorem 1.2 (iv) to the group representation $\alpha \circ P_{\gamma}$ we find that $\sigma\left(\left(\alpha \circ P_{\gamma}\right)_{t}\right)=\{\gamma(t)\}$ for all $t \in G$. It follows from Gelfand's theorem (see [10] or [13]) that $\left(\alpha \circ P_{\gamma}\right)_{t}=\gamma_{0}(t) I_{P_{\gamma} X}$ for all $t \in G$. Clearly, $P_{\gamma} X \neq\{0\}$. 
The following "lifting property" should be compared with a similar result in [15].

Proposition 1.4. Let $\alpha: M(G) \rightarrow \mathcal{L}(X)$ be a bounded group representation. Let $Y \subseteq Z$ be two $\alpha$-invariant closed subspaces of $X$. Assume that $\gamma_{0} \in \operatorname{Sp}(\alpha)$ and $\psi \in(Z / Y)^{*}$ satisfy

$$
\left\langle\psi, \alpha_{t}(z)+Y\right\rangle=\gamma_{0}(t)\langle\psi, z+Y\rangle \quad \text { for all } \quad t \in G, z \in Z .
$$

Then, there exists $x_{0}^{*} \in X^{*}$ such that $\alpha_{t}^{*} x_{0}^{*}=\gamma_{0}(t) x_{0}^{*}$ for all $t \in G$ and $\left\langle x_{0}^{*}, z\right\rangle=$ $\langle\psi, z+Y\rangle$ for all $z \in Z$.

Proof. Define $\psi_{1} \in Z^{*}$ by $\psi_{1}(z):=\psi(z+Y), z \in Z$. By Hahn-Banach theorem we extend $\psi_{1}$ to an element $\psi_{2} \in X^{*}$, such that $\left\|\psi_{2}\right\|=\left\|\psi_{1}\right\|$. Since $G$ is abelian, there exists an invariant mean $\phi$ on $l^{\infty}(G)$. For each $x \in X$, define

$$
F(x ; t):=\gamma_{0}(-t) \psi_{2}\left(\alpha_{t} x\right), \quad t \in G .
$$

This is a function in $l^{\infty}(G)$. It follows that

$$
x_{0}^{*}(x):=\phi(F(x ; \cdot)), \quad x \in X,
$$

well defines a linear functional on $X$ with $\left\|x_{0}^{*}\right\| \leq\|\alpha\| \cdot\|\psi\|$. For $s, t \in G$ and $x \in X$ we have

$$
F\left(\alpha_{s}(x) ; t\right)=\gamma_{0}(-t) \psi_{2}\left(\alpha_{s+t} x\right)=\gamma_{0}(s) F(x ; s+t) .
$$

It follows that

$$
x_{0}^{*}\left(\alpha_{s} x\right)=\gamma_{0}(s) \phi(F(x ; \cdot+s))=\gamma_{0}(s) x_{0}^{*}(x),
$$

where for the last identity we use the translation-invariance of $\phi$. Therefore, $\alpha_{s}^{*} x_{0}^{*}=$ $\gamma_{0}(s) x_{0}^{*}$ for all $s \in G$. Consider $z \in Z$. Then,

$$
F(z ; t)=\gamma_{0}(-t) \psi\left(\alpha_{t}(z)+Y\right)=\gamma_{0}(-t) \gamma_{0}(t) \psi(z+Y)=\psi(z+Y) .
$$

This implies that $x_{0}^{*}(z)=\phi(F(z ; \cdot))=\psi(z+Y) \phi(\mathbf{1})=\psi(z+Y)$, completing the proof.

\section{Completeness of eigenvectors of dual Representations}

Recall that a closed subset $\Lambda$ of $\widehat{G}$ is called scattered if each closed subset of $\Lambda$ contains an isolated point. Every closed countable subset of $\widehat{G}$ is scattered and, moreover, if $\widehat{G}$ satisfies the second axiom of countability, then a closed subset of $\widehat{G}$ is scattered if and only if it is countable.

Our main result in this section reads as follows.

Theorem 2.1. Let $\alpha: G \rightarrow \mathcal{L}(X)$ be an integrable bounded group representation whose Arveson spectrum $\operatorname{Sp}(\alpha)$ is scattered. Then the subspace

$$
\begin{aligned}
X_{\alpha^{*}}:=\operatorname{lin}\left\{x^{*} \in X^{*}: \quad\right. & \text { there exists } \gamma \in \operatorname{Sp}(\alpha) \text { such that } \\
& \left.\alpha_{t}^{*} x^{*}=\gamma(t) x^{*} \text { for all } t \in G\right\}
\end{aligned}
$$

generated by all eigenvectors of $\alpha^{*}$ is $w^{*}$-dense in $X^{*}$. Moreover, there exists a uniformly bounded, mutually orthogonal system of projections $\left\{E_{\gamma}: \gamma \in \operatorname{Sp}(\alpha)\right\}$ such that $E_{\gamma} X^{*}=\left\{x^{*} \in X^{*}: \alpha_{t}^{*} x^{*}=\gamma(t) x^{*}\right.$ for all $\left.t \in G\right\}$ for all $\gamma \in \operatorname{Sp}(\alpha)$. 
Proof. Let $Y:=\left\{y \in X: x^{*}(y)=0\right.$ for all $\left.x^{*} \in X_{\alpha^{*}}\right\}$. Then, $Y$ is an $\alpha$-invariant closed subspace of $X$. We have to show $Y=\{0\}$. To this end, let $\beta_{t}(t \in G)$ be the restriction of $\alpha_{t}$ in $Y$. Then, $\beta$ is also an integrable bounded group representation. By Theorem 1.2 (i), it is sufficient to prove that $\operatorname{Sp}(\beta)=\emptyset$ in order to obtain $Y=\{0\}$.

Assume conversely that $Y \neq\{0\}$. Let $X_{*} \subset X^{*}$ be such that $\alpha$ is $\sigma\left(X, X_{*}\right)$ integrable. Let $Y_{*}:=\left\{x_{\mid Y}^{*}: x^{*} \in Y\right\}$. Then, $\beta$ is $\sigma\left(Y, Y_{*}\right)$ integrable. Let $V$ be the norm closure of $Y_{*}$ in $Y^{*}$. Then, $\beta$ is also integrable with respect to the weak topology $\sigma(Y, V)$. We have $V \neq\{0\}$ and $\beta_{t}^{*} V \subset V$ for each $t \in G$. This implies that

$$
\Phi_{t}:=\text { the restriction of } \beta_{t}^{*} \text { in } V, t \in G,
$$

defines a $\sigma(V, Y)$ integrable bounded group representation. To compute the spectrum of $\Phi$, consider $f \in I_{\alpha}$, i.e., $\tilde{\alpha}_{f}=0$. Then, for all $y \in Y$ and $\rho \in Y_{*}$ we have

$$
\left\langle y, \tilde{\Phi}_{f} \rho\right\rangle=\int_{G}\left\langle y, \Phi_{t} \rho\right\rangle f(t) d t=\int_{G}\left\langle\rho, \alpha_{t} y\right\rangle f(t) d t=\rho\left(\tilde{\alpha}_{f} y\right)=0 .
$$

Therefore, $\tilde{\Phi}_{f}=0$ and thus $I_{\Phi} \supseteq I_{\alpha}$. By definition of spectrum we find that $\operatorname{Sp}(\Phi) \subseteq \operatorname{Sp}(\alpha)$. Since $\operatorname{Sp}(\alpha)$ is scattered, so is $\operatorname{Sp}(\Phi)$. The non-empty scattered set $\operatorname{Sp}(\Phi)$ contains an isolated point, $\gamma_{0}$ say. By Proposition $1.3 \gamma_{0}$ is an eigenvalue of $\Phi$. Choose $0 \neq y_{0}^{*} \in V \subseteq Y^{*}$ to be an eigenvector for $\gamma_{0}$. Then, for all $y \in Y$ and $t \in G$ we have

$$
y_{0}^{*}\left(\alpha_{t} y\right)=\Phi_{t} y_{0}^{*}(y)=\gamma_{0}(t) y_{0}^{*}(y) .
$$

Applying Proposition 1.4 to $\alpha$ with the case $Z=\{0\}$ we obtain an extension $0 \neq x_{0}^{*} \in X^{*}$ of $y_{0}^{*}$ such that $\alpha_{t}^{*} x_{0}^{*}=\gamma_{0}(t) x_{0}^{*}$ for all $t \in G$. It follows that $x_{0}^{*} \in X_{\alpha^{*}}$. Hence, we have for all $y \in Y$ that $y_{0}^{*}(y)=x_{0}^{*}(y)=0$, a contradiction.

To show the "Moreover" part, let $\phi$ be an invariant mean on $l^{\infty}(G)$. Let $\gamma \in$ $\operatorname{Sp}(\alpha)$. For each pair $(x, \rho) \in X \times X^{*}$ the function $t \mapsto\left\langle\rho, \gamma(-t) \alpha_{t} x\right\rangle$ belongs to $l^{\infty}(G)$. Thus,

$$
\left\langle E_{\gamma} \rho, x\right\rangle:=\phi_{t}\left(\left\langle\rho, \gamma(-t) \alpha_{t} x\right\rangle, x \in X, \rho \in X^{*},\right.
$$

defines an operator $E_{\gamma}$ on $X^{*}$, where $\phi_{t}$ means that the invariant mean is applied to the corresponding function of $t$. It is evident that $\left\|E_{\gamma}\right\| \leq\|\alpha\|$. The translationinvariance of $\phi$ implies that $\alpha_{t}^{*} E_{\gamma}=\gamma(t) E_{\gamma}$ for all $t \in G$. From this we see further that each $E_{\gamma}$ is a projection. To prove the mutual orthogonality, let $\gamma_{1}, \gamma_{2} \in \operatorname{Sp}(\alpha)$ be two different elements. Clearly, $E_{\gamma_{1}}$ and $E_{\gamma_{2}}$ are commuting. Therefore, for all $t \in G$

$$
\begin{aligned}
\gamma_{1}(t) E_{\gamma_{1}} E_{\gamma_{2}} & =\alpha_{t}^{*} E_{\gamma_{1}} E_{\gamma_{2}}=\alpha_{t}^{*} E_{\gamma_{2}} E_{\gamma_{1}} \\
& =\gamma_{2}(t) E_{\gamma_{2}} E_{\gamma_{1}}=\gamma_{2}(t) E_{\gamma_{1}} E_{\gamma_{2}} .
\end{aligned}
$$

Since $\gamma_{1} \neq \gamma_{2}$, this implies that $E_{\gamma_{1}} E_{\gamma_{2}}=0$. The proof is finished.

Consider a representation $\alpha$ given as in Theorem 2.1 which is $\sigma\left(X, X_{*}\right)$ integrable. Let $W_{\alpha}$ be the $\sigma\left(X, X_{*}\right)$ closed subalgebra of $\mathcal{L}(X)$ generated by all operators $\tilde{\alpha}_{\mu}$ $(\mu \in M(G))$. Assume $T \in W_{\alpha}$. Then, there exists a net $\left(\mu_{i}\right) \subset M(G)$ such that $\tilde{\alpha}_{\mu_{i}} \stackrel{\sigma}{\longrightarrow} T$. For $\gamma \in \operatorname{Sp}(\alpha)$ consider the projection $E_{\gamma}$ given in Theorem 2.1. Fix 
$(x, \rho) \in X \times X_{*}$. Then, from the definition of $\tilde{\alpha}_{\mu_{i}}$ we have

$$
\begin{aligned}
\left\langle E_{\gamma} \rho, \tilde{\alpha}_{\mu_{i}} x\right\rangle & =\int_{G}\left\langle\alpha_{t}^{*} E_{\gamma} \rho, x\right\rangle d \mu_{i}(t) \\
& =\int_{G}\left\langle\gamma(t) E_{\gamma} \rho, x\right\rangle d \mu_{i}(t)=\widehat{\mu_{i}}(\gamma)\left\langle E_{\gamma} \rho, x\right\rangle .
\end{aligned}
$$

By taking limit we find

$$
\left\langle E_{\gamma} \rho, T x\right\rangle=\lim _{i} \widehat{\mu_{i}}(\gamma)\left\langle E_{\gamma} \rho, x\right\rangle .
$$

Since this identity holds for all $x \in X$ and $\rho \in X_{*}$, it follows that the $\operatorname{limit}_{\lim } \widehat{\mu_{i}}(\gamma)$ exists; denoted by $c_{\gamma}$. Then we have $T^{*} E_{\gamma}=c_{\gamma} E_{\gamma}$. Therefore, the subspace generated by all eigenvectors of $T^{*}$ is $w^{*}$-dense in $X^{*}$. As a consequence, the zero operator is the unique nilpotent operator in $W_{\alpha}$ and thus $W_{\alpha}$ is a semisimple Banach algebra.

This has established the following Theorem 2.2 which refines [20, Theorem 7] where the corresponding result is given for weakly continuous representations. Note that our proof is completely different from that of [20].

Theorem 2.2. Let $\alpha: G \rightarrow \mathcal{L}(X)$ be a $\sigma\left(X, X_{*}\right)$ integrable bounded group representation whose Arveson spectrum $\operatorname{Sp}(\alpha)$ is scattered. Then the $\sigma\left(X, X_{*}\right)$ closed subalgebra $W_{\alpha}$ of $\mathcal{L}(X)$ is semisimple.

Moreover, for each $T \in W_{\alpha}$ there exists a set $\left\{c_{\gamma}: \gamma \in \operatorname{Sp}(\alpha)\right\} \subset \mathbb{C}$ such that $T^{*} E_{\gamma}=c_{\gamma} E_{\gamma}$ for all $\gamma \in \operatorname{Sp}(\alpha)$, where $\left\{E_{\gamma}: \gamma \in \operatorname{Sp}(\alpha)\right\}$ is the set of projections given in Theorem 2.1. As a result, the subspace generated by all eigenvectors of $T^{*}$ is $w^{*}$-dense in $X^{*}$.

As consequences of Theorems 2.1 and 2.2 we have:

Corollary 2.3. Let $T \in \mathcal{L}(X)$ be a doubly power bounded operator with countable spectrum $\sigma(T)$. Then the subspace generated by all eigenvectors of $T^{*}$ is $w^{*}$-dense in $X^{*}$. Moreover, the weakly closed subalgebra generated by $T$ is semisimple.

Proof. Let $\alpha: \mathbb{Z} \rightarrow \mathcal{L}(X)$ be the group representation given by

$$
\alpha_{n}:=T^{n} \text { for all } n \in \mathbb{Z} .
$$

$\alpha$ is norm continuous and bounded. Moreover, by Theorem 1.2 (vi) we have $\operatorname{Sp}(\alpha)=$ $\sigma(T)$. Hence, Theorems 2.1 and 2.2 are applicable to $\alpha$ and yields the desired result.

Corollary 2.4. Let $\left(e^{t A}\right)_{t \in \mathbb{R}}$ be a strongly continuous bounded group of operators on $X$ such that the spectrum $\sigma(A)$ of the infinitesimal generator $A$ is countable. Then the subspace generated by all eigenvectors of the dual operator $A^{*}$ is $w^{*}$-dense in $X^{*}$. Moreover, the smallest weakly closed subalgebra of $\mathcal{L}(X)$ containing all operators $e^{t A}(t \in \mathbb{R})$ is semisimple.

Proof. Let $\alpha: \mathbb{R} \rightarrow \mathcal{L}(X)$ be the strongly continuous bounded group representation given by

$$
\alpha_{t}:=e^{t A} \text { for all } t \in \mathbb{R} .
$$

Then $\operatorname{Sp}(\alpha)=i \sigma(A)$ by Theorem 1.2 (vii). Hence $\operatorname{Sp}(\alpha)$ is countable. If $\lambda \in \sigma(A)$ and $x^{*} \in X^{*}$ satisfy $\alpha_{t}^{*} x^{*}=e^{\lambda t} x^{*}$ for all $t \in \mathbb{R}$, then $x^{*}$ belongs to the definition domain of $A^{*}$ and $A^{*} x^{*}=\lambda x^{*}$. Thus, the result follows by using Theorems 2.1 and 2.2 . 
Recall that an operator $T \in \mathcal{L}(X)$ is called hermitian if $\left\|e^{i t T}\right\|=1$ for all $t \in \mathbb{R}$. From Corollary 2.4 we derive immediately the following analogue of Corollary 2.3.

Corollary 2.5. Let $T \in \mathcal{L}(X)$ be a hermitian operator with countable spectrum $\sigma(T)$. Then the subspace generated by all eigenvectors of $T^{*}$ is $w^{*}$-dense in $X^{*}$. Moreover, the weakly closed subalgebra generated by $T$ is semisimple.

We remark that the semisimplicity in Corollary 2.3 and Corollary 2.5 has been proved by Feldman [9] and Sinclair [22, Theorem 3.1], respectively. Their methods are completely different from that of [20] and ours.

\section{Completeness of eigenvectors}

The completeness of eigenvectors of integrable group representations with discrete spectrum is guaranteed by the following result.

Theorem 3.1. Let $\alpha: G \rightarrow \mathcal{L}(X)$ be an $\sigma\left(X, X_{*}\right)$ integrable bounded representation with discrete Arveson spectrum. Then the subspace

$$
\begin{array}{r}
X_{\alpha}:=\operatorname{lin}\{x \in X: \quad \text { there exists } \gamma \in \operatorname{Sp}(\alpha) \text { such that } \\
\left.\alpha_{t} x=\gamma(t) x \text { for all } t \in G\right\}
\end{array}
$$

generated by all eigenvectors of $\alpha$ is $\sigma\left(X, X_{*}\right)$ dense in $X$. Moreover, there exists a uniformly bounded, mutually orthogonal system of projections $\left\{P_{\gamma}: \gamma \in \operatorname{Sp}(\alpha)\right\}$ such that $P_{\gamma} X=\left\{x \in X: \alpha_{t} x=\gamma(t) x\right.$ for all $\left.t \in G\right\}$ for all $\gamma \in \operatorname{Sp}(\alpha)$.

Proof. Applying Proposition 1.3 to $\alpha$ we find that for each $\gamma \in \operatorname{Sp}(\alpha)$ there exists a projection $P_{\gamma} \in\left\{\tilde{\alpha}_{f}: f \in L^{1}(G)\right\}$ satisfying

$$
\alpha_{t} P_{\gamma}=\gamma(t) P_{\gamma} \text { for all } t \in G \text {. }
$$

Thus,

$$
X_{\alpha}=\bigvee_{\gamma \in \operatorname{Sp}(\alpha)} P_{\gamma} X
$$

Consider $f \in K(G)$, i.e., $\hat{f}$ has compact support. Let $X_{f}:=\overline{\tilde{\alpha}_{f} X}$ and $\beta$ be the restriction of $\alpha$ in $X_{f}$. Then, $\beta$ is integrable and

$$
\operatorname{Sp}(\beta) \subseteq \operatorname{Sp}(\alpha) \cap \operatorname{supp} \hat{f} .
$$

Note that the set $\operatorname{Sp}(\alpha) \cap \operatorname{supp} \hat{f}$ is compact and discrete; hence it contains at most finitely many points. Therefore, $\operatorname{Sp}(\beta)$ is a finite subset of $\operatorname{Sp}(\alpha)$. It follows from Theorem 1.2 (iii) combining with Proposition 1.3 that $X_{f}$ is decomposed into finite sum of eigenspaces of $\beta$. Thus, $X_{f}$ is contained in $X_{\alpha}$. By Theorem $1.2(\mathrm{v})$, the subspace generated by all of the subsets $X_{f}(f \in K(G))$ is $\sigma\left(X, X_{*}\right)$ dense in $X$. Consequently, $X_{\alpha}$ is $\sigma\left(X, X_{*}\right)$ dense in $X$.

A more general result of Theorem 3.1 is given in [14, Theorem 3.1.2].

In what follows we will study the almost periodicity of representations. Let $B U C(G ; X)$ be the Banach space of all bounded and uniformly continuous functions $h: G \rightarrow X$ with the norm

$$
\|h\|:=\sup _{t \in G}\|h(t)\|_{X}
$$

For each $t \in G$ let $T_{t}$ be the translation on $B U C(G ; X)$ given by

$$
T_{t} h(\cdot):=h(\cdot+t) \text { for all } h \in B U C(G ; X) .
$$


A function $h \in B U C(G ; X)$ is called almost periodic if the set $\left\{T_{t} h: t \in G\right\}$ is relatively compact in $B U C(G ; X)$. Let $A P(G ; X)$ be the subspace of all almost periodic functions in $B U C(G ; X)$ and denote by $A P(G)$ the space $A P(G ; \mathbb{C})$. A function $h \in B U C(G ; X)$ is called scalar almost periodic if for each $x^{*} \in X^{*}$ the scalar function $t \mapsto x^{*}(h(t))$ is almost periodic. Let $h \in B U C(G ; X)$. It is well known that $h \in A P(G ; X)$ if and only if $h$ is scalar almost periodic and the range $\{h(t): t \in G\}$ is relatively compact in $X$ (cf. [19, pp. 70-72]).

The Beurling spectrum of $h \in B U C(G ; X)$, denoted by $\sigma(h)$, is defined to be the local spectrum of $T$ at $h$, i.e.,

$$
\sigma(h):=\operatorname{Sp}_{T}(h)=\left\{\gamma \in \widehat{G}: f \in L^{1}(G), \tilde{T}_{f} h=0 \Longrightarrow \hat{f}(\gamma)=0\right\} .
$$

If $h \in B U C(G ; \mathbb{C})$ has scattered spectrum, then a theorem of Loomis [17, Theorem $5]$ asserts that $h$ is almost periodic. The extension of Loomis's theorem to vectorvalued functions is given by Baskakov [2, Theorem 2] as follows. Recall that $c_{0}$ is the Banach space of all convergent sequences $\left(a_{n}\right)_{n \in \mathbb{N}}$ with limit zero.

Theorem 3.2. Assume that $h \in B U C(G ; X)$ has scattered spectrum. If $X$ does not contain any copy of $c_{0}$, then $h$ is almost periodic.

We call a strongly continuous bounded group representation $\alpha: G \rightarrow \mathcal{L}(X)$ almost periodic if for each $x \in X$ the function $t \mapsto \alpha_{t} x$ is almost periodic. It follows from the Jocob-deLeeuw-Glicksberg theory that the subspace generated by all eigenvectors of an almost periodic representation is norm dense in the defining Banach space, see [6, Theorem 4.11], [16] or the Basic Theorem in [18, p.150].

Let $\alpha: G \rightarrow \mathcal{L}(X)$ be a $\sigma\left(X, X_{*}\right)$ integrable bounded group representation whose Arveson spectrum is scattered. Assume further that the Banach space $X$ does not contain any copy of $c_{0}$. For $f \in K(G)$, let $X_{f}:=\overline{\tilde{\alpha}_{f} X}$ and let $\beta$ be the restriction of $\alpha$ in $X_{f}$. As seen in the proof of Theorem 3.1, we have

$$
\operatorname{Sp}(\beta) \subseteq \operatorname{Sp}(\alpha) \cap \operatorname{supp} \hat{f} .
$$

Hence, the representation $\beta$ has compact spectrum and thus is norm continuous by Theorem 1.2 (ii). Given $x_{0} \in X_{f}$, let

$$
h(t):=\beta_{t} x_{0}=\alpha_{t} x_{0}, \quad t \in G .
$$

Then, the norm continuity of $\beta$ implies that $h \in B U C\left(G ; X_{f}\right)$. Let $g \in L^{1}(G)$. For $s \in G$ we have

$$
\tilde{T}_{g} h(s)=\int_{G} T_{t} h(s) g(t) d t=\int_{G} h(s+t) g(t) d t=\int_{G} \alpha_{s+t} x_{0} g(t) d t=\alpha_{s} \tilde{\alpha}_{g} x_{0} .
$$

It follows that $\tilde{T}_{g} h=0 \Longleftrightarrow \tilde{\alpha}_{g} x_{0}$. By definition we find

$$
\sigma(h)=\operatorname{Sp}_{\alpha}\left(x_{0}\right) \subseteq \operatorname{Sp}(\alpha) .
$$

Therefore, $h$ has scattered spectrum. It follows from Theorem 3.1 that $h$ is almost periodic. By definition, $\beta$ is almost periodic and thus by the Jacob-deLeeuwGlicksberg theory [6] the subspace generated by all eigenvectors of $\beta$ is norm dense in $X_{f}$. Note that the subspace generated by the subspaces $X_{f}(f \in K(G))$ is $\sigma\left(X, X_{*}\right)$ dense in $X$ by Theorem $1.2(\mathrm{v})$. We have proved the following result.

Theorem 3.3. Let $\alpha: G \rightarrow \mathcal{L}(X)$ be a $\sigma\left(X, X_{*}\right)$ integrable bounded group representation whose Arveson spectrum $\operatorname{Sp}(\alpha)$ is scattered. Assume that $X$ does not 
contain any copy of $c_{0}$. Then the subspace

$$
\begin{array}{r}
X_{\alpha}:=\operatorname{lin}\{x \in X: \quad \text { there exists } \gamma \in \operatorname{Sp}(\alpha) \text { such that } \\
\left.\alpha_{t} x=\gamma(t) x \text { for all } t \in G\right\}
\end{array}
$$

generated by all eigenvectors of $\alpha$ is $\sigma\left(X, X_{*}\right)$ dense in $X$.

As a result, if $\alpha$ is strongly continuous, then $\alpha$ is almost periodic.

The following refines Corollaries 2.3, 2.4 and 2.5.

Corollary 3.4. Assume that $X$ does not contain any copy of $c_{0}$. Then

(i) If $T \in \mathcal{L}(X)$ is a doubly power bounded operator with countable spectrum $\sigma(T)$, then the subspace generated by all eigenvectors of $T$ is norm dense in $X$.

(ii) If $\left(e^{t A}\right)_{t \in \mathbb{R}}$ is a strongly continuous bounded group of operators on $X$ such that the spectrum $\sigma(A)$ is countable, then the subspace generated by all eigenvectors of $A$ is norm dense in $X$.

(iii) If $T \in \mathcal{L}(X)$ is a hermitian operator with countable spectrum $\sigma(T)$, then the subspace generated by all eigenvectors of $T$ is norm dense in $X$.

\section{REFERENCES}

[1] Arveson, W., On groups of automorphisms of operator algebras. J. Funct. Anal. 15 (1974), 217-243. MR 50:1016

[2] Baskakov, A.G., Spectral criteria for almost periodicity of solutions of functional equations. Math. Notes of Acad. Sci. USSR 24 (1978), 606-612.

[3] Bratteli, O. and Robinson, D.W., Operator Algebras and Quantum Statistical Mechanics I. Springer-Verlag, New York-Heidelberg-Berlin (1979). MR 81a:46070

[4] Connes, A., Une classification des facteurs de type III. Ann. Sci. l'École Norm. Sup. 6 (1973), 133-245. MR 49:5865

[5] Corojonră, I. and Foiaş, C., Theory of Generalized Spectral Operators. Gordon and Breach, New York, 1968. MR 52:15085

[6] deleeuw, K. and Glicksberg, L., Applications of almost periodic compactifications. Acta Math. 105 (1961), 63-97. MR 24:A1632

[7] D'Antoni, C., Longo, C., And Zsidó, L., A spectral mapping theorem for locally compact groups of operators. Pacific J. Math. 103 (1982), 17-24. MR 84e:47058

[8] Dugundur, J., Topology, 4th. ed. Allyn and Bacon Inc., Boston, 1968.

[9] Feldman, G.M., The semisimplicity of an algebra generated by an isometric operator, Funct. Anal. Appl. 8(1974), 93-94. MR 50:14245

[10] Gelfand, I.M., Ideale und primäre Ideale in normierten Ringen. Mat. Sb. 9 (1941), 41-47.

[11] Hewitt, E. AND Ross, R., Abstract Harmonic Analysis I. Springer-Verlag, BerlinHeidelberg-New York, 1963. MR 28:158

[12] Hille, E. And Phillips, R. S., Functional Analysis and Semi-Groups, 3rd ed. Rhode Island, Amer. Math. Soc. Colloq. Publ. XXXI, 1968. MR 54:11077

[13] Huang, S.-Z., Characterizing spectra of closed operators through existence of slowly growing solutions of their Cauchy problems. Studia Math. 116 (1995), 23-41. MR 96i:47068

[14] Huang, S.-Z., Spectral Theory for Non-Quasianalytic Representations of Locally Compact Abelian Groups. Thesis, Universität Tübingen, 1996. A complete summary is given in "Dissertation Summaries in Mathematics" 1 (1996), 171-178.

[15] Huang, S.-Z., van Neerven, J. and RäBiger, F., Ditkin's condition for certain Beurling algebras. Proc. Amer. Math. Soc. 126 (1998), 1397-1407. CMP 97:11

[16] Krengel, U., Ergodic Theorems. de Gruyter, Berlin, New York (1985). MR 87i:28001

[17] Loomis, L. H., The spectral characterization of a class of almost periodic functions. Ann. Math. 72 (1960), 362-368. MR 22:11255

[18] LyUbich, Yu. I., Introduction to the Theory of Banach Representations of Groups. Birkhäuser-Verlag, Basel (1988). MR 90i:22001

[19] Levitan, B.M. and Zhikov, V.V., Almost Periodic Functions and Differential Equations. Cambridge Univ. Press, Cambridge (1982). MR 84g:34004 
[20] Muraz, G. And VŨ, Quôc Phóng, Semisimple Banach algebras generated by strongly continuous representations of locally compact abelian groups. J. Funct. Anal. 126 (1994), 1-6. MR 95k:43006

[21] Rudin, W., Fourier Analysis on Groups. Interscience, New York, 1962. MR 27:2808

[22] Sinclair, A.M., The Banach algebra generated by a hermitian operator, Proc. London Math. Soc. 24 (1972), 681-691. MR 46:4198

Mathematisches Institut, Friedrich-Schiller-Universität Jena, Ernst-Abbe-Platz 1-4, D-07743 Jena, Germany

Current address: Fachbereich Mathematik, Universität Rostock, Universitätsplatz 1, 18055 Rostock, Germany

E-mail address: huang@sun.math.uni-rostock.de 